\title{
Hyperbolic User Interfaces for Computer Aided Architectural Design
}

\author{
Volkmar Hovestadt ${ }^{*}$ and Oliver Gramberg ${ }^{* *}$ and Oliver Deussen ${ }^{* *}$ \\ ${ }^{*}$ Institut für Industrielle Bauproduktion, ${ }^{* *}$ Institut für Betriebs- und Dialogsysteme \\ University of Karlsruhe \\ 76128 Karlsruhe, Germany \\ phone: $++49 / 721 / 608-2167$ \\ volkmar@ifib1.ifib.uni-karlsruhe.de
}

\begin{abstract}
We present basic ideas and a prototypical implementation of a graphical user interface intended for the architectural design process. The interface integrates CAD-like object manipulation and navigation through large data sets. Navigation and working area are not separated as done in prior work. Geometric transformations produce a deformed but controllable display of the data in the border area of the working window while normal working is possible in the untransformed center. The arrangement allows the user to shift directly from construction tasks to navigation tasks. Data reduction techniques have been implemented in order to reduce the cost of transformation and display.
\end{abstract}

KEYWORDS: Computer Aided Architectural Design (CAAD), Information Visualization, Fisheye Technique, Focus+Context Technique, Interaction

Architectural design processes tend to integrate models isolated so far. Many different kinds of expert knowledge, tools, visualization techniques, and media are to be combined. The design process covers the complete life cycle of the building, i. e. construction as well as operation, reorganization, and destruction. Considering the shared use of digital design tools and the exchange of information and knowledge between designers and across different projects, we speak of a design continuиm.

An architect's work involves mostly visually represented data. Problems are often outlined and dealt with in a graphical manner. Only this form of expression serves as a basis for work and discussion.

Therefore the designer should have maximum visual control over the processes taking place within the design continuum. Further questions arise about navigation, associative information access, programming and communication within very large data sets.

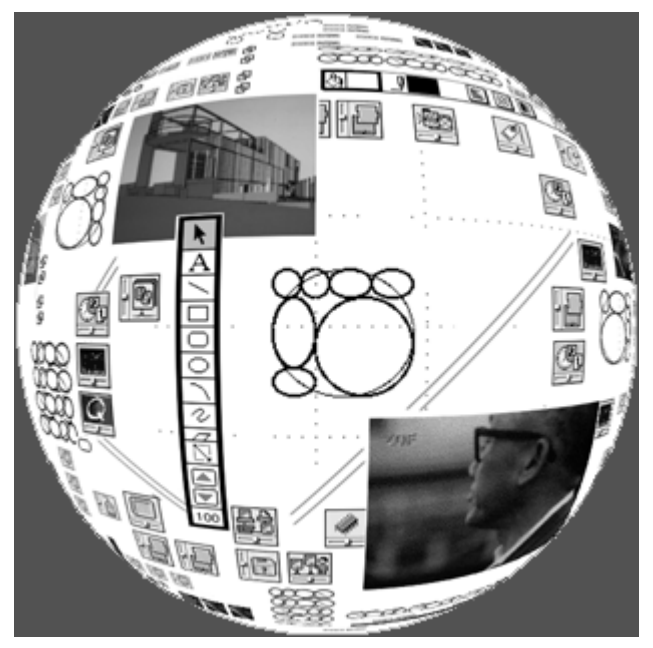

Figure 1: Scenario of the proposed user interface

First we look for a mapping of the design continuum onto a limited working area, e. g. a computer screen. We seek to maximize the amount of information visible to and manageable by the user. In addition to known methods like the use of scrollbars or overall views the literature contains diverse proposals of display techniques $([1,4,2])$. We are concerned not only in the transformation of possibly unconstrained data in order to achieve better navigation and control, but also in the full integration of a working area into the transformed data display and thereby a continuous transition from design to navigation.

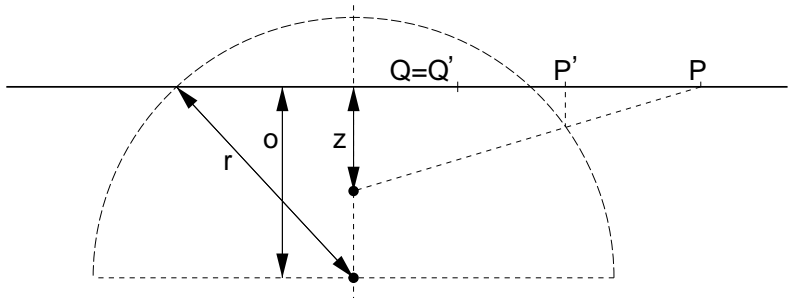

Figure 2: Transformation process

Transformation of the data in the object plane is accomplished by central projection onto a so-called base corpus and, in a second step, by parallel projection back onto the object plane. 


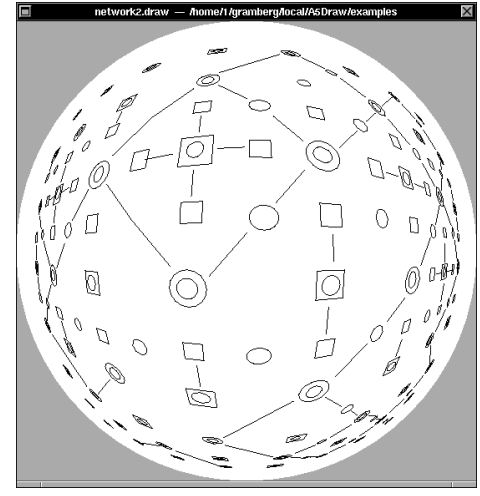

(a)

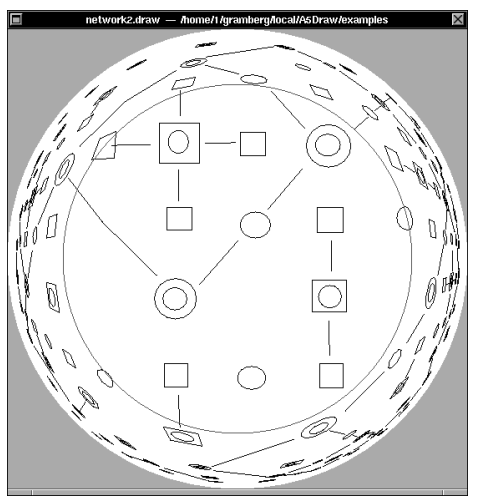

(b)

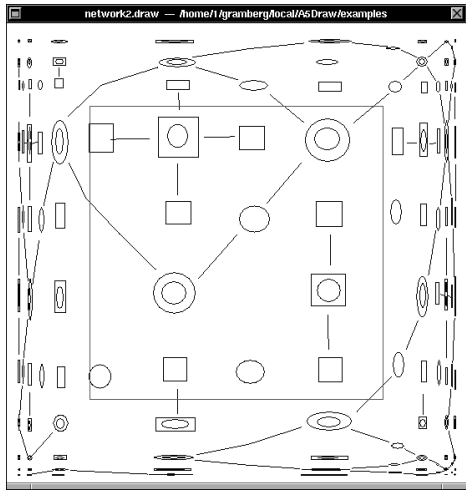

(c)

Figure 3: (a) spherical projection (b) projection with untransformed area (c) coordinate-wise transformation

Hyperboloides, cones, and spheres have been considered for base corpuses. In addition to the bivariate transformation also a coordinate-wise transformation has been implemented which preserves straight lines parallel to the axes (Fig. 3.)

The transformation is termed hyperbolic because the size of objects converges to zero with growing distance from the point of view. This allows possibly infinite data planes to be transformed into a finite display.

In the case of a spherical base corpus the mapping function of the $x$-coordinate is

$$
\tilde{x}=x \frac{\sqrt{\left(x^{2}+z^{2}\right)\left(r^{2}-(z-o)^{2}\right)+z^{2}(z-o)^{2}}+z(z-o)}{x^{2}+z^{2}}
$$

where $x$ is the distance of the point in the object plane to be transformed from the perpendicular of the center of the sphere onto the plane, $r$ is the radius of the sphere, $z$ is the distance between the projection center and the object plane, and $o$ is the distance between the center of the sphere and the object plane (cf. Fig. 2).

All transformations divide the interface into two parts. The center shows an undistorted portion of the data where objects are displayed at full size and complexity and can be manipulated with tools commonly available in CAD applications. This working area is framed by a transformed display of the other data (cf. Fig. 3.)

The division for a spherical base corpus is obtained by the following case distinction:

$$
x^{\prime}= \begin{cases}\tilde{x} & x<\sqrt{r^{2}-o^{2}} \\ x & \text { otherwise }\end{cases}
$$

We regard the combination of the working area and the overview/navigation instrument as an advantage over existing navigation aids, because it permits the visually-working architect to switch smoothly between object editing and navigation. Efforts like lenses ([3]) have been considered, but they do not provide this kind of transition between the planar and the transformed parts of the data as desired by the application.

Navigation within the partially extremely distorted display is possible because the graphical data generated by architectu- ral design processes commonly show predominant structures which allow recognition even under extreme deformation. All transformations can be parameterized in order to alter the display of the data from a large working area with a thin and highly distorted border to a small working area with a broad and less distorted border. Combination with zooming effects is possible. A compressed overall view is just a special case of our transformation.

The work on the different transformation methods has been accompanied by the development of techniques for reduction of the visual data. The representation of the data outside the working area is possibly modified by two methods. Complex objects like text fragments, pictures, or free-hand drawings are being replaced by their bounding box. Different kinds of objects are discernable by different colors. Objects with transformed sizes below a given threshold are being drawn as dots. The interface was realized so far under NeXTStep with Postscript, ObjectiveC and C.

The efficiency of the implemented methods with respect to user requirements has been verified by a series of tests involving simulation of the behaviour of an architect using the design environment.

\section{REFERENCES:}

[1] Furnas, G. W.: Generalized Fisheye Views, in Proc. CHI'86 Human Factors in Computing Systems, 1988, ACM Press, pp. 16-23

[2] Mackinlay, J. D., and Robertson, G. G., and Card, S. K.: The Perspective Wall: Detail and Context Smoothly Inegrated, in Proc. CHI'92 Human Factors in Computing Systems, 1991, ACM Press, pp. 173-179

[3] Rao, R., and Card, S. K.: The Table Lens: Merging Graphical and Symbolic Representations in an Interactive Focus+Contex Visualization for Tabular Information, in Proc. CHI'94 Human Factors in Computing Systems, 1994, ACM Press

[4] Sarkar, M., and Brown, M.: Graphical Fisheye Views of Graphs, in Proc. CHI'92 Human Factors in Computing Systems, 1992, ACM Press, pp. 83-91 